\title{
Existence and Continuous Dependence on Initial Data of Solution for Initial Value Problem of Fuzzy Multiterm Fractional Differential Equation
}

\author{
Huichol Choi, ${ }^{1}$ Kinam Sin $\mathbb{D}^{1},{ }^{1}$ Sunae Pak, ${ }^{1}$ and Sungryol So ${ }^{2}$ \\ ${ }^{1}$ Faculty of Mathematics, Kim Il Sung University, Pyongyang, Democratic People's Republic of Korea \\ ${ }^{2}$ Institute of Advanced Science, Kim Il Sung University, Pyongyang, Democratic People's Republic of Korea \\ Correspondence should be addressed to Kinam Sin; 15bf12025@hit.edu.cn
}

Received 17 March 2019; Revised 13 May 2019; Accepted 15 May 2019; Published 19 June 2019

Academic Editor: Rustom M. Mamlook

Copyright (C) 2019 Huichol Choi et al. This is an open access article distributed under the Creative Commons Attribution License, which permits unrestricted use, distribution, and reproduction in any medium, provided the original work is properly cited.

In this paper, the fuzzy multiterm fractional differential equation involving Caputo-type fuzzy fractional derivative of order $0<\alpha<$ 1 is considered. The uniqueness of solution is established by using the contraction mapping principle and the existence of solution is obtained by Schauder fixed point theorem.

\section{Introduction}

Nowadays the fractional differential equations (FDEs) are powerful tools representing many problems in various areas such as control engineering, diffusion processes, signal processing, and electromagnetism.

Recently the fuzzy fractional differential equations (FFDEs) have been studied by many researchers in order to analyze some systems with fuzzy initial conditions. But, it is too difficult to find the exact solutions of most FFDEs representing real-world phenomena. Therefore, research about FFDEs can be classified into two classes, namely, existence of solution and numerical methods. Many theoretical researches have been advanced on the existence, uniqueness, and stability of solution of FFDEs [1-12].

Also the analytical method and the numerical method are typical methods for solving FFDEs. The analytical method includes the Laplace transform method, monotone iterative method, variation of constant formula, and so on [13-15]. Typical numerical methods are the operational matrix method, fractional Euler method, predictor-corrector method, and so on [16-21].
In [22], the existence and uniqueness of the solutions of fuzzy initial value problems of fractional differential equations with the Caputo-type fuzzy fractional derivative have been proved. Under the conditions which the right sides of equations satisfy Hölder continuity or Lipschitz continuity in its all variables, the existence of a solution to the Cauchy problem for fuzzy fractional differential equations was discussed in [23]. In [24], by employing the contraction mapping principle on the complete metric space, the existence and uniqueness result for fuzzy fractional functional integral equation has been proved. The existence results of solutions for fuzzy fractional initial value problem under generalized differentiability conditions are obtained by Banach fixed point theorem in [25]. In [26], researchers discussed the uniqueness and existence of the solutions for FFDEs with Riemann-Liouville H-differentiability of arbitrary order by using Krasnoselskii-Krein type conditions, Kooi type conditions, and Rogers conditions. But, the considerations of researchers in [22-26] were restricted to the case of FFDEs with single derivative term. Ngo et al. [27] presented that the existence and uniqueness results of the solution for fuzzy Caputo-Katugampola (CK) fractional differential equations with initial value and in [28] proved that the fractional 
fuzzy differential equation is not equal to the fractional fuzzy integral equation in general.

Based on the above facts, in this paper, we study the existence and uniqueness of solutions for fuzzy multiterm fractional differential equations of order $0<\alpha<1$ with fuzzy initial value under Caputo-type $\mathrm{H}$-differentiability.

The paper is organized as follows. In Section 2, we introduced some definitions and properties of fuzzy fractional calculus. The existence result of solution for proposed problem is described in Section 3. Section 4 presented the continuous dependence on initial data of solution. Finally, the conclusion is summarized in Section 5.

\section{Preliminaries and Basic Results}

We introduce some definitions and notations which will be used throughout our paper.

Definition 1 (see [29]). Let us denote by $\mathbf{R}_{\mathrm{F}}$ the class of fuzzy subsets $u: \mathbf{R} \longrightarrow[0,1]$ satisfying the following properties:

(i) $u$ is normal, i.e., $\exists x_{0} \in \mathbf{R}$ for which $u\left(x_{0}\right)=1$,

(ii) $u$ is fuzzy convex, i.e., $u(\lambda x+(1-\lambda) y) \geq$ $\min \{u(x), u(y)\}$ for all $\forall x, y \in \mathbf{R}, \forall \lambda \in[0,1]$,

(iii) $u$ is upper semicontinuous on $\mathbf{R}$,

(iv) supp $u=\{x \in \mathbf{R} \mid u(x)>0\}$ is the support of the $u$, and its closure $\mathrm{cl}(\operatorname{supp} u)$ is compact.

Then $\mathbf{R}_{\mathrm{F}}$ is called the space of fuzzy number and any $u \in$ $\mathbf{R}_{\mathrm{F}}$ is called fuzzy number.

We denote the $r$-cut form of fuzzy number $u \in \mathbf{R}_{\mathrm{F}}, 0 \leq$ $r \leq 1$, by $[u]^{r}:=\left[u_{1}(r), u_{2}(r)\right]$.

Also let us $u, v \in \mathbf{R}_{\mathrm{F}}$. The metric $d: \mathbf{R}_{\mathrm{F}} \times \mathbf{R}_{\mathrm{F}} \longrightarrow[0,+\infty)$ on $\mathbf{R}_{\mathrm{F}}$ is defined as follows:

$$
\begin{aligned}
& d(u, v) \\
& \quad:=\sup _{r \in[0,1]} \max \left\{\left|u_{1}(r)-v_{1}(r)\right|,\left|u_{2}(r)-v_{2}(r)\right|\right\} .
\end{aligned}
$$

Definition 2 (see [19]). Let $u, v \in \mathbf{R}_{\mathrm{F}}$. If there exists $w \in \mathbf{R}_{\mathrm{F}}$ such that $u=v \oplus w$, then $w$ is called the H-difference of $u u$ and $v$, and it is denoted by $w=u \Theta_{H} v$. Note that $u \Theta_{H} v \neq$ $u+(-1) v$.

Definition 3 (see [19]). Let $f:(a, b) \longrightarrow \mathbf{R}_{\mathrm{F}}$ and $x_{0} \in(a, b)$. We say that $f$ is H-differentiable at $x_{0}$, if for $h>0$ sufficiently near to 0 , there exist the H-differences $f\left(x_{0}+h\right) \Theta_{H} f\left(x_{0}\right)$, $f\left(x_{0}\right) \Theta_{H} f\left(x_{0}-h\right)$, and the limits

$$
\begin{aligned}
& \lim _{h \rightarrow 0^{+}} \frac{f\left(x_{0}+h\right) \Theta_{H} f\left(x_{0}\right)}{h} \\
& \quad=\lim _{h \rightarrow 0^{+}} \frac{f\left(x_{0}\right) \Theta_{H} f\left(x_{0}-h\right)}{h} .
\end{aligned}
$$

Then the limit is denoted by $D^{(1)} f\left(x_{0}\right)$.
Theorem 4 (see [14]). Let $f:[a, b] \longrightarrow \mathbf{R}_{\mathrm{F}}$ be H-differentiable and $[f(x)]^{r}:=\left[f_{1}(x, r), f_{2}(x, r)\right]$. Then $f_{1}(x, r), f_{2}(x, r)$ are all differentiable and

$$
\left[D^{(1)} f(x)\right]^{r}=\left[f_{1}^{\prime}(x, r), f_{2}^{\prime}(x, r)\right] .
$$

Definition 5 (see [14]). A function $f:[a, b] \longrightarrow \mathbf{R}_{\mathrm{F}}$ is said to be Riemann integrable on $[a, b]$, if $\exists I_{R} \in \mathbf{R}_{\mathrm{F}}, \forall \varepsilon>0, \exists \delta>0$, for any division of $[a, b], \Delta: a=t_{0}<t_{1}<\cdots<t_{n}=b$ with norm $\lambda(\Delta)<\delta$, and for any points $\xi_{i} \in\left[t_{i}, t_{i+1}\right], i=$ $0,1, \ldots, n-1$,

$$
d\left(\sum_{i=0}^{n-1} f\left(\xi_{i}\right) \cdot\left(t_{i+1}-t_{i}\right), I_{R}\right)<\varepsilon .
$$

We denote the fuzzy Riemann integral of $f$ from $a$ to $b$ by $I_{R}=\int_{a}^{b} f(t) d t$.

Lemma 6 (see [14]). Suppose that $f, g:[a, b] \rightarrow \mathbf{R}_{\mathrm{F}}$ are continuous, then

(i) $f$ is the fuzzy Riemann integrable on $[a, b]$ and $F(t)=$ $\int_{a}^{t} f(s) d s$ is differentiable as in Definition 3, namely, $D^{(1)} F(t)=$ $f(t)$.

(ii) $d\left(\int_{a}^{b} f(s) d s, \int_{a}^{b} g(s) d s\right) \leq \int_{a}^{b} d(f(s), g(s)) d s$.

We introduce following notations:

$C^{F}(I)$ is the set of all continuous fuzzy-valued functions on

$I$

$A C^{F}(I)$ is the set of all absolutely continuous fuzzy-valued functions on $I$.

$L^{F}(I)$ is the space of all Lebesque integrable fuzzy-valued functions on $I$, where $I=[0, L]$ and without losing generality, we promise that $L=1$.

Definition 7 (see [19]). Let $f \in C^{F}(I) \cap L^{F}(I)$. The fuzzy Riemann-Liouville fractional integral of the fuzzy-valued function $f$ is defined as follows:

$$
I_{0+}^{\beta} f(x)=\frac{1}{\Gamma(\beta)} \int_{0}^{x}(x-s)^{\beta-1} f(s) d s, \quad x>0 .
$$

where $I_{0+}^{\beta}$ is the Riemann-Liouville integral operator of $\beta$ and $\Gamma(\beta)$ is the Gamma function.

Lemma 8 (see [19]). Let $f \in C^{F}(I) \cap L^{F}(I)$. Then RiemannLiouville integral of the fuzzy-valued function $f$, based on its $r$-cut form, can be expressed as follows:

$$
\left[I_{0+}^{\beta} f(x)\right]^{r}=\left[I_{0+}^{\beta} f_{1}(x, r), I_{0+}^{\beta} f_{2}(x, r)\right], \quad 0 \leq r \leq 1,
$$

where

$$
I_{0+}^{\beta} f_{i}(x, r):=\frac{1}{\Gamma(\beta)} \int_{0}^{x} \frac{f_{i}(s, r)}{(x-s)^{1-\beta}} d s, \quad i=1,2 .
$$

Lemma 9. Let $f \in C^{F}(I), \alpha, \beta>0$. Then the following relations are satisfied:

$$
I_{0+}^{\alpha} I_{0+}^{\beta} f(x)=I_{0+}^{\beta} I_{0+}^{\alpha} f(x)=I_{0+}^{\alpha+\beta} f(x) .
$$


Proof. Let us denote the $r$-cut form of $f$ by $[f(x)]^{r}:=$ $\left[f_{1}(x, r), f_{2}(x, r)\right]$.

Then we have

$$
\begin{aligned}
{\left[I_{0+}^{\alpha} I_{0+}^{\beta} f(x)\right]^{r} } & =\left[I_{0+}^{\alpha} I_{0+}^{\beta} f_{1}(x, r), I_{0+}^{\alpha} I_{0+}^{\beta} f_{2}(x, r)\right] \\
& =\left[I_{0+}^{\beta} I_{0+}^{\alpha} f_{1}(x, r), I_{0+}^{\beta} I_{0+}^{\alpha} f_{2}(x, r)\right] \\
& =\left[I_{0+}^{\beta} I_{0+}^{\alpha} f(x)\right]^{r} .
\end{aligned}
$$

Moreover, since $f \in C^{F}(I)$, we get

$$
\begin{aligned}
{\left[I_{0+}^{\alpha} I_{0+}^{\beta} f(x)\right]^{r} } & =\left[I_{0+}^{\alpha+\beta} f_{1}(x, r), I_{0+}^{\alpha+\beta} f_{2}(x, r)\right] \\
& =\left[I_{0+}^{\alpha+\beta} f(x)\right]^{r} .
\end{aligned}
$$

Definition 10 (see [19]). Let $0<\beta<1$. We say that $f$ is fuzzy Riemann-Liouville $\mathrm{H}$-differentiable of order $\beta$ if

$$
I_{0+}^{1-\beta} f(x)=\frac{1}{\Gamma(1-\beta)} \int_{0}^{x} \frac{f(s)}{(x-s)^{\beta}} d s, \quad x>0
$$

is $\mathrm{H}$-differentiable. Then fuzzy Riemann-Liouville $\mathrm{H}$ derivative of order $\beta$ of function $f$ is denoted by ${ }^{R L} D_{0+}^{\beta} f(x):=D^{(1)} I_{0+}^{1-\beta} f(x)$.

Definition 11 (see [19]). Let $0<\beta<1$. We say that $f$ is a fuzzy Caputo-type differentiable function if $\mathrm{H}$-difference $f(x) \Theta_{H} f(0)$ exists and $I_{0+}^{1-\beta}\left(f(x) \Theta_{H} f(0)\right) \in A C^{F}(I)$ satisfies. Then fuzzy Caputo-type derivative of order $\beta$ of function $f$ is denoted by

$$
\left({ }^{c} D_{0+}^{\beta} f\right)(x):={ }^{R L} D_{0+}^{\beta}\left(f(x) \oplus_{H} f(0)\right), \quad x>0 .
$$

Lemma 12 (see [19]). Let $f \in C^{F}(I) \cap L^{F}(I)$ and $[f(x)]^{r}:=$ $\left[f_{1}(x, r), f_{2}(x, r)\right]$ for $\forall r \in[0,1]$. If $f$ is a fuzzy Caputo-type fractional differentiable function, then

$$
\begin{aligned}
{\left[\left({ }^{c} D_{0+}^{\beta} f\right)(x)\right]^{r} } & =\left[\left(I_{0+}^{1-\beta} D f\right)(x)\right]^{r} \\
& =\left[I_{0+}^{1-\beta} D f_{1}(x, r), I_{0+}^{1-\beta} D f_{2}(x, r)\right] .
\end{aligned}
$$

Lemma 13. Let $f:[a, b] \longrightarrow \mathbf{R}_{\mathrm{F}}$ be H-differentiable. Then the following relations hold:

(i) $f \in C^{F}(0,1] \Longrightarrow{ }^{c} D_{0+}^{\beta} I_{0+}^{\beta} f(x)=f(x)$.

(ii) $f \in C^{F}(0,1] \Longrightarrow{ }^{R L} D_{0+}^{\beta} I_{0+}^{\beta} f(x)=f(x)$.

(iii) $0<\lambda<\beta, f \in C^{F}(0,1] \Longrightarrow{ }^{c} D_{0+}^{\lambda} I_{0+}^{\beta} f(x)=$ $I_{0+}^{\beta-\lambda} f(x)$.

Proof. First we prove (i). From the assumption of Lemma 13, we have

$$
\begin{aligned}
{ }^{c} D_{0+}^{\beta} I_{0+}^{\beta} f(x) & ={ }^{R L} D_{0+}^{\beta}\left(I_{0+}^{\beta} f(x) \Theta_{H} I_{0+}^{\beta} f(0)\right) \\
& =D^{(1)} I_{0+}^{1-\beta}\left(I_{0+}^{\beta} f(x) \Theta_{H} I_{0+}^{\beta} f(0)\right), \\
& =D^{(1)} I_{0+}^{1-\beta}\left(I_{0+}^{\beta} f(x)\right)=D^{(1)} I_{0+}^{1} f(x) .
\end{aligned}
$$

By Lemma 6 (i), we get

$$
D^{(1)} I_{0+}^{1} f(x)=f(x) .
$$

From the result (i) of lemma, it is obvious that (ii) holds. Next let prove (iii).

$$
\begin{aligned}
{ }^{c} D_{0+}^{\lambda} I_{0+}^{\beta} f(x) & ={ }^{R L} D_{0+}^{\lambda}\left(I_{0+}^{\beta} f(x) \Theta_{H} I_{0+}^{\beta} f(0)\right) \\
& =D^{(1)} I_{0+}^{1-\lambda}\left(I_{0+}^{\beta} f(x) \Theta_{H} I_{0+}^{\beta} f(0)\right) \\
& =D^{(1)} I_{0+}^{1-\lambda}\left(I_{0+}^{\beta} f(x)\right) \\
& =D^{(1)} I_{0+}^{1+\beta-\lambda} f(x) .
\end{aligned}
$$

By Lemma 6 (i), we obtain

$$
D^{(1)} I_{0+}^{1+\beta-\lambda} f(x)=I_{0+}^{\beta-\lambda} f(x) .
$$

Lemma 14. The following facts are true:

(i) Let $z \in C^{F}(I)$. For any positive number $\sigma$, the fractional integral $I_{0+}^{\sigma} z(x)$ is continuous in $x$.

(ii) For any positive numbers $\sigma$ and $k$, it holds that $I_{0+}^{\sigma} e^{k x} \leq$ $e^{k x} / k^{\alpha}$. that

(iii) Let $u, v \in C^{F}(I)$. For any positive number $\sigma$, it holds

$$
d\left(I_{0+}^{\sigma} u(x), I_{0+}^{\sigma} v(x)\right) \leq I_{0+}^{\sigma} d(u(x), v(x)) .
$$

Proof. Let us consider the assertion (i). For any $x_{0} \in(0,1]$, it is enough to prove that

$$
\lim _{x \longrightarrow x_{0}} d\left(I_{0+}^{\sigma} z(x), I_{0+}^{\sigma} z\left(x_{0}\right)\right)=0 .
$$

We use the notation $[z(x)]^{r}:=\left[z_{1}(x, r), z_{2}(x, r)\right]$ for $r$-cut representation of $z(x)$.

Since $z \in C^{F}(I), z_{1}(r, x)$, and $z_{2}(r, x)$ are continuous in $x$. And by Lemma 8 , the following expression holds:

$$
\begin{aligned}
d & \left(I_{0+}^{\sigma} z(x), I_{0+}^{\sigma} z\left(x_{0}\right)\right) \\
& =\sup _{r \in[0,1]} \max \left\{\left|I_{0+}^{\sigma} z_{1}(x, r)-I_{0+}^{\sigma} z_{1}\left(x_{0}, r\right)\right|,\right. \\
& \left.\left|I_{0+}^{\sigma} z_{2}(x, r)-I_{0+}^{\sigma} z_{2}\left(x_{0}, r\right)\right|\right\} .
\end{aligned}
$$

It is well known that $I_{0+}^{\sigma} z_{i}(x, r), i=1,2$, are continuous with respective to $x$. Thus we can see that $\lim _{x \rightarrow x_{0}} d\left(I_{0+}^{\sigma} z(x), I_{0+}^{\sigma} z\left(x_{0}\right)\right)=0$.

Now we prove (ii). 
From the definition of fractional integral, the following is true:

$$
\begin{aligned}
I_{0+}^{\alpha} e^{k t} & =\frac{1}{\Gamma(\alpha)} \int_{0}^{t}(t-s)^{\alpha-1} e^{k s} d s \\
& =\frac{1}{\Gamma(\alpha)} \int_{0}^{t} z^{\alpha-1} e^{k(t-z)} d z=\frac{e^{k t}}{\Gamma(\alpha)} \int_{0}^{t} z^{\alpha-1} e^{-k t} d z \\
& =\frac{e^{k t}}{\Gamma(\alpha)} \int_{0}^{k t}\left(\frac{s}{k}\right)^{\alpha-1} e^{-s} \frac{1}{k} d s \\
& =\frac{e^{k t}}{\Gamma(\alpha) \cdot k^{\alpha}} \cdot \int_{0}^{k t} s^{\alpha-1} e^{-s} d s
\end{aligned}
$$

By the definition of Gamma function, we have

$$
\leq \frac{e^{k t}}{\Gamma(\alpha) \cdot \mathrm{k}^{\alpha}} \cdot \int_{0}^{\infty} s^{\alpha-1} e^{-s} d s=\frac{e^{k t}}{\Gamma(\alpha) \cdot \mathrm{k}^{\alpha}} \Gamma(\alpha)=\frac{e^{k t}}{\mathrm{k}^{\alpha}} .
$$

Let us consider assertion (iii).

We use the notations $[u(x)]^{r}:=\left[u_{1}(x, r), u_{2}(x, r)\right]$, $[v(x)]^{r}:=\left[v_{1}(x, r), v_{2}(x, r)\right]$ for $r$-cut representations of $u(x)$, $v(x)$, respectively.

Since $u, v \in C^{F}(I), u_{1}(x, r), u_{2}(x, r), v_{1}(x, r), v_{2}(x, r)$ are continuous in $x$. Therefore the following evaluations are true:

$$
\begin{aligned}
& d\left(I_{0+}^{\sigma} u(x), I_{0+}^{\sigma} v(x)\right) \\
& =\sup _{r \in[0,1]} \max \left\{\left|I_{0+}^{\sigma} u_{1}(x, r)-I_{0+}^{\sigma} v_{1}(x, r)\right|,\right. \\
& \left.\left|I_{0+}^{\sigma} u_{2}(x, r)-I_{0+}^{\sigma} v_{2}(x, r)\right|\right\} \\
& \quad=\sup _{r \in[0,1]} \max \left\{\left|I_{0+}^{\sigma}\left(u_{1}(x, r)-v_{1}(x, r)\right)\right|,\right. \\
& \left.\left|I_{0+}^{\sigma}\left(u_{2}(x, r)-v_{2}(x, r)\right)\right|\right\} \\
& \leq \sup _{r \in[0,1]} \max \left\{I_{0+}^{\sigma}\left|u_{1}(x, r)-v_{1}(x, r)\right|,\right. \\
& \left.I_{0+}^{\sigma}\left|u_{2}(x, r)-v_{2}(x, r)\right|\right\} \\
& \leq I_{0+}^{\sigma} \sup _{r \in[0,1]} \max \left\{\left|u_{1}(x, r)-v_{1}(x, r)\right|,\right. \\
& \left.\left|u_{2}(x, r)-v_{2}(x, r)\right|\right\}=I_{0+}^{\sigma} d\left(z_{1}(x), z_{2}(x)\right) .
\end{aligned}
$$

The proof is completed.

\section{Existence of Solution for Fuzzy Multiterm Fractional Differential Equation}

Let us consider the existence of solution for initial value problem of following fuzzy multiterm fractional differential equation:

$$
\begin{aligned}
{ }^{c} D_{0+}^{\alpha} y(x) & =f\left(x, y(x),{ }^{c} D_{0+}^{\beta} y(x)\right), \quad x \in(0,1], \\
y(0) & =y_{0}, \\
0 & <\beta<\alpha<1,
\end{aligned}
$$

where $f: I \times \mathbf{R}_{\mathrm{F}} \times \mathbf{R}_{\mathrm{F}} \longrightarrow \mathbf{R}_{\mathrm{F}}, y_{0} \in \mathbf{R}_{\mathrm{F}}$, and ${ }^{c} D_{0+}^{\alpha}$ is fuzzy Caputo-type derivative.

Definition 15. Let $y: I \longrightarrow \mathbf{R}_{\mathrm{F}}$. We say that $y$ is the solution of initial value problem (24) if ${ }^{c} D_{0+}^{\beta} y(x) \in C^{F}(I)$ holds and $y$ satisfies (24).

Now let us consider the following:

$$
\begin{aligned}
{ }^{c} D_{0+}^{\alpha} y(x) & =z(x), \quad x \in(0,1], \\
y(0) & =y_{0}, \\
y_{0} & \in \mathbf{R}_{\mathrm{F}},
\end{aligned}
$$

where $z(x) \in C^{F}(I)$.

Lemma 16. The solution of initial value problem of fuzzy fractional differential equation (25) is represented as

$$
y(x)=y_{0} \oplus \frac{1}{\Gamma(\alpha)} \int_{0}^{x} \frac{z(s)}{(x-s)^{1-\alpha}} d s .
$$

Proof. Let $y$ be the solution of initial value problem (25). Then we have

$$
{ }^{c} D_{0+}^{\alpha} y(x) \equiv z(x), \quad x \in I .
$$

Also since $z$ is the fuzzy continuous, it is the fuzzy integrable and $I_{0+}^{\alpha} z(x)$ exists for $x \in I$.

Therefore the following relations hold:

$$
\begin{aligned}
I_{0+}^{\alpha c} D_{0+}^{\alpha} y(x) & \equiv I_{0+}^{\alpha} z(x), \quad x \in I, \\
I_{0+}^{\alpha}{ }^{R L} D_{0+}^{\alpha}\left(y(x) \Theta_{H} y_{0}\right) & \equiv I_{0+}^{\alpha} z(x), \\
I_{0+}^{\alpha} D^{(1)} I_{0+}^{1-\alpha}\left(y(x) \Theta_{H} y_{0}\right) & \equiv I_{0+}^{\alpha} z(x) .
\end{aligned}
$$

From the Caputo-type differentiability of fuzzy-valued function $y$, we get

$$
I_{0+}^{1-\alpha}\left(y(x) \Theta_{H} y_{0}\right) \in A C^{F}(I)
$$

and since the space of the absolutely continuous functions coincides with the space of primitive functions of Lebesque integrable functions, the following relation is satisfied:

$$
\begin{aligned}
\exists \varphi & \in L^{F}(I) ; \\
I_{0+}^{1-\alpha}\left(y(x) \Theta_{H} y_{0}\right) & =I_{0+}^{1} \varphi(x) .
\end{aligned}
$$

Therefore we obtain

$$
y(x) \Theta_{H} y_{0}=I_{0+}^{\alpha} \varphi(x) .
$$

By (30) and Lemma 6 (i), the left side of (28) exchanges as

$$
\begin{aligned}
& I_{0+}^{\alpha} D^{(1)} I_{0+}^{1-\alpha}\left(y(x) \Theta_{H} y_{0}\right)=I_{0+}^{\alpha} D^{(1)} I_{0+}^{1} \varphi(x) \\
& \quad=I_{0+}^{\alpha} \varphi(x) .
\end{aligned}
$$

From (28) and (31), $y(x) \Theta_{H} y_{0}=I_{0+}^{\alpha} z(x)$ is satisfied. Namely, we have

$$
y(x)=y_{0} \oplus I_{0+}^{\alpha} z(x)
$$


Conversely, we prove that $y$ denoted by (26) is the solution of fuzzy initial value problem (25). Since

$$
y(x)=y_{0} \oplus \frac{1}{\Gamma(\alpha)} \int_{0}^{x} \frac{z(s)}{(x-s)^{1-\alpha}} d s=y_{0} \oplus I_{0+}^{\alpha} z(x),
$$

$y(x) \Theta_{H} y_{0}=I_{0+}^{\alpha} z(x)$ holds. Also as $z \in C^{F}(I)$, we get

$$
I_{0+}^{1-\alpha}\left(y(x) \Theta_{H} y_{0}\right)=I_{0+}^{1} z(x) .
$$

On the other hand, when $[z(x)]^{r}:=\left[z_{1}(x, r), z_{2}(x, r)\right]$, by Lemma 8

$$
\left[I_{0+}^{1} z(x)\right]^{r}=\left[\int_{0}^{x} z_{1}(s, r) d s, \int_{0}^{x} z_{2}(s, r) d s\right]
$$

holds $\forall r \in[0,1]$. Therefore

$$
\begin{aligned}
& {\left[I_{0+}^{1} z(x+h)\right]^{r}=\left[\int_{0}^{x+h} z_{1}(s, r) d s, \int_{0}^{x+h} z_{2}(s, r) d s\right]} \\
& \quad=\left[\int_{0}^{x} z_{1}(s, r) d s\right. \\
& \left.\quad+\int_{x}^{x+h} z_{1}(s, r) d s, \int_{0}^{x} z_{2}(s, r) d s+\int_{x}^{x+h} z_{2}(s, r) d s\right] \\
& \quad=\left[\int_{0}^{x} z_{1}(s, r) d s, \int_{0}^{x} z_{2}(s, r) d s\right] \\
& +\left[\int_{x}^{x+h} z_{1}(s, r) d s, \int_{x}^{x+h} z_{2}(s, r) d s\right] .
\end{aligned}
$$

Since the interval family $\left\{\left[\int_{x}^{x+h} z_{1}(s, r) d s, \int_{x}^{x+h} z_{2}(s\right.\right.$, $r) d s]\}_{r}$ generates obviously a fuzzy number, we can see that there exist the $\mathrm{H}$-differences as

$$
\begin{aligned}
& I_{0+}^{1} z(x+h) \Theta_{H} I_{0+}^{1} z(x), \\
& I_{0+}^{1} z(x) \Theta_{H} I_{0+}^{1} z(x-h) .
\end{aligned}
$$

Consequently the H-differentiability of $y$ is leaded.

From (35), we obtain

$$
D^{(1)} I_{0+}^{1-\alpha}\left(y(x) \Theta_{H} y_{0}\right)=D^{(1)} I_{0+}^{1} z(x)=z(x) .
$$

Also it is obvious that $y$ satisfies the initial condition.

Theorem 17. Let $f$ of (24) be a fuzzy continuous with respect to every variable. If $y(x)$ is the solution of initial value problem (24), the fuzzy-valued function $z(x)$ which is constructed by $z(x):={ }^{c} D_{0+}^{\alpha} y(x)$ is the solution in $C^{F}(I)$ of fuzzy integral equation as

$$
\begin{array}{r}
z(x) \\
=f\left(x, y_{0} \oplus \frac{1}{\Gamma(\alpha)} \int_{0}^{x} \frac{z(s)}{(x-s)^{1-\alpha}} d s, I_{0+}^{\alpha-\beta} z(x)\right), \\
x \in(0,1] .
\end{array}
$$

Conversely if $z(x)$ is the solution in $C^{F}(I)$ of fuzzy integral equation (40), $y(x)$ which is constructed by (26) is the solution of initial value problem (24).
Proof. Let $y(x)$ be the solution of initial value problem (24). Namely, let assume that $y(x)$ satisfies

$$
\begin{aligned}
{ }^{c} D_{0+}^{\alpha} y(x) & =f\left(x, y(x),{ }^{c} D_{0+}^{\beta} y(x)\right), \quad x \in(0,1], \\
y(0) & =y_{0}, \\
y_{0} & \in \mathbf{R}_{\mathrm{F}} .
\end{aligned}
$$

Then if $z(x)$ is denoted by $z(x):={ }^{c} D_{1}^{\alpha} y(x)$, by Lemma 16 , the following relation is leaded:

$$
y(x)=y_{0} \oplus \frac{1}{\Gamma(\alpha)} \int_{0}^{x} \frac{z(s)}{(x-s)^{1-\alpha}} d s=y_{0} \oplus I_{0+}^{\alpha} z(x) .
$$

Applying the operator ${ }^{c} D_{0+}^{\beta}$ to the both side of above equation, by Lemma 13 (iii), we get

$$
{ }^{c} D_{0+}^{\beta} y(x)=I_{0+}^{\alpha-\beta} z(x) .
$$

Therefore substituting the above results to ${ }^{c} D_{0+}^{\alpha} y(x)$, ${ }^{c} D_{0+}^{\beta} y(x), y(x)$ of (41), we obtain (40).

Next let $z(x)$ be the solution of fuzzy integral equation (40). Then it is obvious that

$$
y(x)=y_{0} \oplus I_{0+}^{\alpha} z(x)
$$

satisfies the initial condition of problem (24) from the continuousness of $z(x)$.

Namely

$$
y_{0}=y(0) .
$$

Consequently from (44)

$$
y(x) \Theta_{H} y_{0}=y(x) \Theta_{H} y(0)=I_{0+}^{\alpha} z(x)
$$

is leaded and regarding the above equation, we get

$$
\begin{aligned}
& I_{0+}^{1-\alpha}\left(y(x) \Theta_{H} y(0)\right)=I_{0+}^{1-\alpha} I_{0+}^{\alpha} z(x)=I_{0+}^{1} z(x), \\
& D^{(1)} I_{0+}^{1-\alpha}\left(y(x) \Theta_{H} y(0)\right)=D^{(1)} I_{0+}^{1} z(x)=z(x), \\
& { }^{c} D_{0+}^{\alpha} y(x)=z(x)=f\left(x, y(s), I_{0+}^{\alpha-\beta} z(x)\right) .
\end{aligned}
$$

Moreover since

$$
\begin{aligned}
{ }^{c} D_{0+}^{\beta} y(x) & ={ }^{c} D_{0+}^{\beta}\left(I_{0+}^{\alpha} z(x) \oplus y(0)\right)={ }^{c} D_{0+}^{\beta} I_{0+}^{\alpha} z(x) \\
& =I_{0+}^{\alpha-\beta} z(x),
\end{aligned}
$$

we obtain

$$
{ }^{c} D_{0+}^{\alpha} y(x)=f\left(x, y(s),{ }^{c} D_{0+}^{\beta} y(x)\right) .
$$

Now we employ the following metric structure in $C^{F}(I)$ :

$$
\begin{aligned}
\forall u, v & \in C^{F}(I), \\
d^{*}(u, v) & :=\max _{t \in I} d(u(t), v(t)) .
\end{aligned}
$$


Obviously we can see that $\left(C^{F}(I), d^{*}\right)$ is a complete metric space (see [24]).

For any positive number $k$, we can consider the metric structure as

$$
\begin{gathered}
\forall u, v \in C^{F}(I), \\
d_{k}^{*}(u, v):=\max _{t \in I} e^{-k t} d(u(t), v(t)) .
\end{gathered}
$$

Then the metric $d_{k}^{*}$ is equivalent to the metric $d^{*}$. Namely,

$$
\begin{aligned}
\exists M, m & >0 ; \\
\forall u, v & \in C^{F}(I), \\
m d_{k}^{*}(u, v) & \leq d^{*}(u, v) \leq M d_{k}^{*}(u, v) .
\end{aligned}
$$

Theorem 18. Assume that the function $f$ in (40) is continuous in its all variables and especially, for any $y_{1}, y_{2}, z_{1}, z_{2} \in \mathbf{R}_{\mathrm{F}}, f$ satisfies the following condition:

$$
\begin{aligned}
& d\left(f\left(x, y_{1}, z_{1}\right), f\left(x, y_{2}, z_{2}\right)\right) \\
& \quad \leq L_{1} \cdot d\left(y_{1}, y_{2}\right)+L_{2} \cdot d\left(z_{1}, z_{2}\right) .
\end{aligned}
$$

Then the fuzzy integral equation (40) has a unique solution.

Proof. Since $\alpha, \alpha-\beta>0$, there exists $k_{*}>0$ that inequality $L_{1}\left(1 / k_{*}{ }^{\alpha}\right)+L_{2}\left(1 / k_{*}{ }^{\alpha-\beta}\right)<1$ is true.

Therefore for any $k_{*}$ satisfying this inequality, we put as follows:

$$
q:=L_{1} \frac{1}{k_{*}^{\alpha}}+L_{2} \frac{1}{k_{*}{ }^{\alpha-\beta}} .
$$

Also we define the operator $T$ by

$$
\begin{aligned}
& (T z)(x) \\
& \quad:=f\left(x, y_{0} \oplus \frac{1}{\Gamma(\alpha)} \int_{0}^{x} \frac{z(s)}{(x-s)^{1-\alpha}} d s, I_{0+}^{\alpha-\beta} z(x)\right) .
\end{aligned}
$$

For any $z \in C^{F}(I)$, by (i) of Lemma $14, I_{0+}^{\alpha} z(x), I_{0+}^{\alpha-\beta} z(x)$ are continuous and $f$ is continuous by assumptions of theorem. Thus the operator $T$ is a map from $C^{F}(I)$ to $C^{F}(I)$.

Thus the fuzzy integral equation (40) is represented as

$$
z=T z, \quad z \in C^{F}(I)
$$

The existence of solution for the fuzzy integral equation (40) is equivalent to the existence of the fixed point of the operator $T$ in $C^{F}(I)$.
For any $z_{1}, z_{2} \in C^{F}(0,1]$, let us evaluate $d\left(T z_{1}(x)\right.$, $T z_{2}(x)$ ).

$$
\begin{aligned}
& d\left(T z_{1}(x), T z_{2}(x)\right)=d\left(f \left(x, y_{0}\right.\right. \\
& \left.\oplus \frac{1}{\Gamma(\alpha)} \int_{0}^{x} \frac{z_{1}(s)}{(x-s)^{1-\alpha}} d s, I_{0+}^{\alpha-\beta} z_{1}(x)\right), f\left(x, y_{0}\right. \\
& \left.\left.\oplus \frac{1}{\Gamma(\alpha)} \int_{0}^{x} \frac{z_{2}(s)}{(x-s)^{1-\alpha}} d s, I_{0+}^{\alpha-\beta} z_{2}(x)\right)\right) \leq L_{1} d\left(y_{0}\right. \\
& \oplus \frac{1}{\Gamma(\alpha)} \int_{0}^{x} \frac{z_{1}(s)}{(x-s)^{1-\alpha}} d s, y_{0} \oplus \frac{1}{\Gamma(\alpha)} \\
& \left.\cdot \int_{0}^{x} \frac{z_{2}(s)}{(x-s)^{1-\alpha}} d s\right)+L_{2} \cdot d\left(I_{0+}^{\alpha-\beta} z_{1}(x),\right. \\
& \left.I_{0+}^{\alpha-\beta} z_{2}(x)\right) \leq L_{1} d\left(\frac{1}{\Gamma(\alpha)} \int_{0}^{x} \frac{z_{1}(s)}{(x-s)^{1-\alpha}} d s, \frac{1}{\Gamma(\alpha)}\right. \\
& \left.\cdot \int_{0}^{x} \frac{z_{2}(s)}{(x-s)^{1-\alpha}} d s\right)+L_{2} \cdot d\left(I_{0+}^{\alpha-\beta} z_{1}(x),\right. \\
& \left.I_{0+}^{\alpha-\beta} z_{2}(x)\right)
\end{aligned}
$$

By (iii) of Lemma 14, we have

$$
\begin{aligned}
\leq & L_{1} \frac{1}{\Gamma(\alpha)} \int_{0}^{x} \frac{d\left(z_{1}(s), z_{2}(s)\right)}{(x-s)^{1-\alpha}} d s+L_{2} \\
& \cdot I_{0+}^{\alpha-\beta} d\left(z_{1}(x), z_{2}(x)\right) \\
= & L_{1} \frac{1}{\Gamma(\alpha)} \int_{0}^{x} \frac{e^{-k_{*} s} e^{k_{*} s} d\left(z_{1}(s), z_{2}(s)\right)}{(x-s)^{1-\alpha}} d s+L_{2} \\
& \cdot I_{0+}^{\alpha-\beta} e^{-k_{*} x} e^{k_{*} x} d\left(z_{1}(x), z_{2}(x)\right) \\
\leq & L_{1} \frac{1}{\Gamma(\alpha)} \int_{0}^{x} \frac{e^{k_{*} s}}{(x-s)^{1-\alpha}} d s \cdot d_{k_{*}}^{*}\left(z_{1}, z_{2}\right)+L_{2} \\
& \cdot\left(I_{0+}^{\alpha-\beta} e^{k_{*} s}\right) \cdot d_{k_{*}}^{*}\left(z_{1}, z_{2}\right)
\end{aligned}
$$

By (ii) of Lemma 14, we have

$$
\leq\left(L_{1} \frac{e^{k_{*} x}}{k_{*}^{\alpha}}+L_{2} \cdot \frac{e^{k_{*} x}}{k_{*}{ }^{\alpha-\beta}}\right) \cdot d_{k_{*}^{*}}^{*}\left(z_{1}, z_{2}\right) .
$$

Consequently the following inequality is obtained:

$$
\begin{aligned}
d\left(T z_{1}(x), T z_{2}(x)\right) \leq & \left(L_{1} \frac{e^{k_{*} x}}{k_{*}{ }^{\alpha}}+L_{2} \cdot \frac{e^{k_{*} x}}{k_{*}{ }^{\alpha-\beta}}\right) \\
& \cdot d_{k_{*}^{*}}^{*}\left(z_{1}, z_{2}\right) .
\end{aligned}
$$

Multiplying the both sides of the above equation by $e^{-k_{*} x}$, we obtain that

$$
\begin{aligned}
& e^{-k_{*} x} d\left(T z_{1}(x), T z_{2}(x)\right) \\
& \quad \leq\left(L_{1} \frac{1}{k_{*}^{\alpha}}+L_{2} \cdot \frac{1}{k_{*}^{\alpha-\beta}}\right) \cdot d_{k_{*}^{*}}^{*}\left(z_{1}, z_{2}\right)
\end{aligned}
$$


and

$$
\begin{aligned}
d_{k_{*}}^{*}\left(T z_{1}, T z_{2}\right) \leq & \left(L_{1} \frac{1}{k_{*}^{\alpha}}+L_{2} \cdot \frac{1}{k_{*}^{\alpha-\beta}}\right) \\
& \cdot d_{k_{*}}^{*}\left(z_{1}, z_{2}\right)=q \cdot d_{k_{*}}^{*}\left(z_{1}, z_{2}\right)
\end{aligned}
$$

Thus the operator $T$ is contractive on $C^{F}(I)$ with respect to the distance $d_{k_{*}^{*}}^{*}$ and we obtain the unique fixed point $C^{F}(I)$ of the operator $T$ by contraction mapping principle. By the way, since the distance $d_{k_{*}}^{*}$ is equivalent to $d^{*}$ in $C^{F}(I), z^{*}$ is also the unique fixed point in sense of the distance $d^{*}$. This completes the proof of theorem.

Next let us consider the existence of solution in case which have not satisfied the Lipschitz condition.

Lemma 19 (Schauder fixed point theorem). Assume that $(E, d)$ is the complete metric space, $U$ is a nonempty convex closed subset of $E$, and $A$ is a continuous mapping of $U$ into itself such that $A(U)$ is contained in a compact subset of $U$, then A has a fixed point in $U$.

Theorem 20. Suppose that the followings conditions are satisfied:

(i) $\exists r>d^{*}\left(f\left(\cdot, y_{0}, \widehat{0}\right), y_{0}\right), L_{1}, L_{2}, L_{3}>0, \forall x_{1}, x_{2} \in$ $(0,1], \forall y_{1}, y_{2} \in U_{r}\left(y_{0}\right), \forall z_{1}, z_{2} \in U_{r}(\widehat{0})$,

$$
\begin{aligned}
& d\left(f\left(x_{1}, y_{1}, z_{1}\right), f\left(x_{2}, y_{2}, z_{2}\right)\right) \\
& \quad \leq L_{1} \cdot d\left(y_{1}, y_{2}\right)+L_{2} \cdot d\left(z_{1}, z_{2}\right)+L_{3} \cdot\left|x_{1}-x_{2}\right|,
\end{aligned}
$$

where $U_{r}\left(y_{0}\right):=\left\{u \mid d^{*}\left(u, y_{0}\right) \leq r, u \in C^{F}(I)\right\}$ and $\widehat{0}$ is zero fuzzy number.

$$
\begin{aligned}
& \text { (ii) } q^{*}:=L_{1}(1 / \Gamma(\alpha+1))+L_{2} \cdot 1 / \Gamma(\alpha-\beta+1)<1 . \\
& \text { Let } \\
& x_{*}:=\max \left\{x \mid L_{1} \cdot x^{\alpha}+L_{2} \cdot x^{\alpha-\beta}\right. \\
& \quad \leq \frac{\min \{\Gamma(\alpha+1), \Gamma(\alpha-\beta+1)\}\left(r-d^{*}\left(f\left(x, y_{0}, \hat{0}\right), y_{0}\right)\right)}{r}, \\
& \left.\frac{x^{\alpha}}{\Gamma(\alpha+1)} \leq 1, \frac{x^{\alpha}}{\Gamma(\alpha-\beta+1)} \leq 1\right\}
\end{aligned}
$$

and

$$
\begin{aligned}
\bar{U}_{r}\left(y_{0}\right):=\left\{u \mid d^{*}\left(u, y_{0}\right) \leq r, u \in C^{F}\left[0, x_{*}\right]\right\}, \\
\bar{U}_{r}(\widehat{0}):=\left\{u \mid d^{*}(u, \widehat{0}) \leq r, u \in C^{F}\left[0, x_{*}\right]\right\} .
\end{aligned}
$$

Then the following results hold:

(i) $T: \bar{U}_{r}(\widehat{0}) \longrightarrow \bar{U}_{r}(\widehat{0})$,

(ii) $\left\{T u \mid u \in \bar{U}_{r}(\widehat{0})\right\}$ is relatively compact.

Proof. Firstly, let us prove that $T: \bar{U}_{r}(\widehat{0}) \longrightarrow \bar{U}_{r}(\widehat{0})$.
We have that, for any $u \in \bar{U}_{r}(\widehat{0})$,

$$
\begin{aligned}
& d\left(y_{0} \oplus \frac{1}{\Gamma(\alpha)} \int_{0}^{x} \frac{u(s)}{(x-s)^{1-\alpha}} d s, y_{0}\right) \\
& \leq d\left(\frac{1}{\Gamma(\alpha)} \int_{0}^{x} \frac{u(s)}{(x-s)^{1-\alpha}} d s, \widehat{0}\right) \\
& \leq \frac{1}{\Gamma(\alpha)} \int_{0}^{x} \frac{d(u(s), \widehat{0})}{(x-s)^{1-\alpha}} d s \leq \frac{x^{\alpha} d^{*}(u, \widehat{0})}{\Gamma(\alpha+1)} \leq r .
\end{aligned}
$$

Thus we can get that $y_{0} \oplus(1 / \Gamma(\alpha)) \int_{0}^{x} u(s) /(x-s)^{1-\alpha} d s \epsilon$ $\bar{U}_{r}\left(y_{0}\right)$.

Also by (iii) of Lemma 14, we have that, for any $u \in \bar{U}_{r}(\widehat{0})$,

$$
\begin{aligned}
d\left(I_{0+}^{\alpha-\beta} u(x), \widehat{0}\right) & \leq I_{0+}^{\alpha-\beta} d(u(x), \widehat{0}) \\
& \leq \frac{x^{\alpha-\beta}}{\Gamma(\alpha-\beta+1)} d^{*}(u(x), \widehat{0}) \leq r .
\end{aligned}
$$

Thus we can see that $I_{0+}^{\alpha-\beta} u(x) \in \bar{U}_{r}(\widehat{0})$.

Therefore we can get that for any $u \in \bar{U}_{r}(\widehat{0})$,

$$
\begin{aligned}
& d\left(T u(x), y_{0}\right)=d\left(f \left(x, y_{0}\right.\right. \\
& \left.\left.\oplus \frac{1}{\Gamma(\alpha)} \int_{0}^{x} \frac{u(s)}{(x-s)^{1-\alpha}} d s, I_{0+}^{\alpha-\beta} u(x)\right), y_{0}\right) \\
& \quad \leq d\left(f \left(x, y_{0}\right.\right. \\
& \left.\left.\quad \frac{1}{\Gamma(\alpha)} \int_{0}^{x} \frac{u(s)}{(x-s)^{1-\alpha}} d s, I_{0+}^{\alpha-\beta} u(x)\right), f\left(x, y_{0}, \widehat{0}\right)\right) \\
& +d\left(f\left(x, y_{0}, \widehat{0}\right), y_{0}\right) \leq L_{1} \cdot d\left(y_{0} \oplus \frac{1}{\Gamma(\alpha)}\right. \\
& \left.\quad \cdot \int_{0}^{x} \frac{u(s)}{(x-s)^{1-\alpha}} d s, y_{0}\right)+L_{2} \cdot d\left(I_{0+}^{\alpha-\beta} u(x), \widehat{0}\right) \\
& \quad+d\left(f\left(x, y_{0}, \widehat{0}\right), y_{0}\right) \leq L_{1} \cdot d\left(\frac{1}{\Gamma(\alpha)}\right. \\
& \left.\quad+\int_{0}^{x} \frac{u(s)}{(x-s)^{1-\alpha}} d s, \widehat{0}\right)+L_{2} \cdot d\left(I_{0+}^{\alpha-\beta} u(x), \widehat{0}\right) \\
& \quad d\left(f\left(x, y_{0}, \widehat{0}\right), y_{0}\right)
\end{aligned}
$$


By (iii) of Lemma 14, we have

$$
\begin{aligned}
\leq & L_{1} \frac{1}{\Gamma(\alpha)} \int_{0}^{x} \frac{d(u(s), \widehat{0})}{(x-s)^{1-\alpha}} d s+L_{2} \cdot I_{0+}^{\alpha-\beta} d(u(x), \widehat{0}) \\
& +d\left(f\left(x, y_{0}, \widehat{0}\right), y_{0}\right) \\
\leq & \frac{L_{1} \cdot x^{\alpha} \cdot r}{\Gamma(\alpha+1)}+\frac{L_{2} \cdot x^{\alpha-\beta} \cdot r}{\Gamma(\alpha-\beta+1)}+d\left(f\left(x, y_{0}, \widehat{0}\right), y_{0}\right) \\
\leq & \frac{L_{1} \cdot x_{*}^{\alpha} \cdot r}{\min \{\Gamma(\alpha+1), \Gamma(\alpha-\beta+1)\}} \\
& +\frac{L_{2} \cdot x_{*}^{\alpha-\beta} \cdot r}{\min \{\Gamma(\alpha+1), \Gamma(\alpha-\beta+1)\}} \\
& +d\left(f\left(x, y_{0}, \widehat{0}\right), y_{0}\right) \leq r .
\end{aligned}
$$

Therefore the operator $T$ is a continuous mapping from convex closed subset $\bar{U}_{r}(\widehat{0})$ into itself; namely,

$$
T: \bar{U}_{r}(\widehat{0}) \longrightarrow \bar{U}_{r}(\widehat{0})
$$

Next let us prove (ii). The uniformly boundness of $\{T u \mid$ $\left.u \in \bar{U}_{r}(\widehat{0})\right\}$ is obvious from (70).

Let us consider the equicontinuity of $\left\{T u \mid u \in \bar{U}_{r}(\widehat{0})\right\}$. For any $y \in\left\{T u \mid u \in \bar{U}_{r}(\widehat{0})\right\}$, there exists $z \in \bar{U}_{r}(\widehat{0})$ which satisfies $y=T z$.

For $x_{1}, x_{2} \in\left(0, x_{*}\right]$, we estimate $d\left(y\left(x_{1}\right), y\left(x_{2}\right)\right)=$ $d\left(T z\left(x_{1}\right), T z\left(x_{2}\right)\right)$.

Without losing generality, let $x_{1}<x_{2} \leq x_{*}$.

Then we have

$$
\begin{aligned}
& d\left(T z\left(x_{1}\right), T z\left(x_{2}\right)\right)=d\left(f \left(x_{1}, y_{0} \oplus \frac{1}{\Gamma(\alpha)}\right.\right. \\
& \left.\quad \cdot \int_{0}^{x_{1}} \frac{z(s)}{\left(x_{1}-s\right)^{1-\alpha}} d s,\left.I_{0+}^{\alpha-\beta} z(x)\right|_{x=x_{1}}\right), \\
& \left.f\left(x_{2}, y_{0} \oplus \frac{1}{\Gamma(\alpha)} \int_{0}^{x_{2}} \frac{z(s)}{\left(x_{2}-s\right)^{1-\alpha}} d s,\left.I_{0+}^{\alpha-\beta} z(x)\right|_{x=x_{2}}\right)\right) \\
& \quad=d\left(f \left(x_{1}, y_{0} \oplus \frac{1}{\Gamma(\alpha)}\right.\right. \\
& \quad \cdot \int_{0}^{x_{1}} \frac{z(s)}{\left(x_{1}-s\right)^{1-\alpha}} d s, \frac{1}{\Gamma(\alpha-\beta)} \\
& \left.\quad \cdot \int_{0}^{x_{1}} \frac{z(s)}{\left(x_{1}-s\right)^{1-(\alpha-\beta)}} d s\right), f\left(x_{2}, y_{0}^{\oplus} \frac{1}{\Gamma(\alpha)}\right. \\
& \quad \cdot \int_{0}^{x_{2}} \frac{z(s)}{\left(x_{2}-s\right)^{1-\alpha}} d s, \frac{1}{\Gamma(\alpha-\beta)}
\end{aligned}
$$

$$
\begin{aligned}
& \left.\left.\cdot \int_{0}^{x_{2}} \frac{z(s)}{\left(x_{2}-s\right)^{1-(\alpha-\beta)}} d s\right)\right) \leq d\left(f \left(x_{1}, y_{0}\right.\right. \\
& \oplus \frac{1}{\Gamma(\alpha)} \int_{0}^{x_{1}} \frac{z(s)}{\left(x_{1}-s\right)^{1-\alpha}} d s, \frac{1}{\Gamma(\alpha-\beta)} \\
& \left.\cdot \int_{0}^{x_{1}} \frac{z(s)}{\left(x_{1}-s\right)^{1-(\alpha-\beta)}} d s\right), f\left(x_{2}, y_{0} \oplus \frac{1}{\Gamma(\alpha)}\right. \\
& \cdot \int_{0}^{x_{1}} \frac{z(s)}{\left(x_{1}-s\right)^{1-\alpha}} d s, \frac{1}{\Gamma(\alpha-\beta)} \\
& \left.\left.\cdot \int_{0}^{x_{1}} \frac{z(s)}{\left(x_{1}-s\right)^{1-(\alpha-\beta)}} d s\right)\right)+d\left(f \left(x_{2}, y_{0}\right.\right. \\
& \oplus \frac{1}{\Gamma(\alpha)} \int_{0}^{x_{1}} \frac{z(s)}{\left(x_{1}-s\right)^{1-\alpha}} d s, \frac{1}{\Gamma(\alpha-\beta)} \\
& \left.\cdot \int_{0}^{x_{1}} \frac{z(s)}{\left(x_{1}-s\right)^{1-(\alpha-\beta)}} d s\right), f\left(x_{2}, y_{0} \oplus \frac{1}{\Gamma(\alpha)}\right. \\
& \cdot \int_{0}^{x_{2}} \frac{z(s)}{\left(x_{2}-s\right)^{1-\alpha}} d s, \frac{1}{\Gamma(\alpha-\beta)} \\
& \left.\left.\cdot \int_{0}^{x_{2}} \frac{z(s)}{\left(x_{2}-s\right)^{1-(\alpha-\beta)}} d s\right)\right) \leq L_{3}\left|x_{1}-x_{2}\right| \\
& +L_{1} d\left(y_{0} \oplus \frac{1}{\Gamma(\alpha)} \int_{0}^{x_{1}} \frac{z(s)}{\left(x_{1}-s\right)^{1-\alpha}} d s, y_{0} \oplus \frac{1}{\Gamma(\alpha)}\right. \\
& \left.\cdot \int_{0}^{x_{2}} \frac{z(s)}{\left(x_{2}-s\right)^{1-\alpha}} d s\right)+L_{2} d\left(\frac{1}{\Gamma(\alpha-\beta)}\right. \\
& \cdot \int_{0}^{x_{1}} \frac{z(s)}{\left(x_{1}-s\right)^{1-(\alpha-\beta)}} d s, \frac{1}{\Gamma(\alpha-\beta)} \\
& \left.\cdot \int_{0}^{x_{2}} \frac{z(s)}{\left(x_{2}-s\right)^{1-(\alpha-\beta)}} d s\right)=L_{3}\left|x_{1}-x_{2}\right| \\
& +L_{1} d\left(\frac{1}{\Gamma(\alpha)} \int_{0}^{x_{1}} \frac{z(s)}{\left(x_{1}-s\right)^{1-\alpha}} d s, \frac{1}{\Gamma(\alpha)}\right. \\
& \left.\cdot \int_{0}^{x_{2}} \frac{z(s)}{\left(x_{2}-s\right)^{1-\alpha}} d s\right)+L_{2} d\left(\frac{1}{\Gamma(\alpha-\beta)}\right. \\
& \cdot \int_{0}^{x_{1}} \frac{z(s)}{\left(x_{1}-s\right)^{1-(\alpha-\beta)}} d s, \frac{1}{\Gamma(\alpha-\beta)} \\
& \left.\cdot \int_{0}^{x_{2}} \frac{z(s)}{\left(x_{2}-s\right)^{1-(\alpha-\beta)}} d s\right) \text {. }
\end{aligned}
$$

Now we estimate the second term of the above expression.

$$
\begin{aligned}
& d\left(\frac{1}{\Gamma(\alpha)} \int_{0}^{x_{1}} \frac{z(s)}{\left(x_{1}-s\right)^{1-\alpha}} d s, \frac{1}{\Gamma(\alpha)} \int_{0}^{x_{2}} \frac{z(s)}{\left(x_{2}-s\right)^{1-\alpha}} d s\right) \\
& \quad=d\left(\frac{1}{\Gamma(\alpha)} \int_{0}^{x_{1}} \frac{z(s)}{\left(x_{1}-s\right)^{1-\alpha}} d s, \frac{1}{\Gamma(\alpha)}\right.
\end{aligned}
$$




$$
\begin{aligned}
& \left.\int_{0}^{x_{1}} \frac{z(s)}{\left(x_{2}-s\right)^{1-\alpha}} d s \oplus \frac{1}{\Gamma(\alpha)} \int_{x_{1}}^{x_{2}} \frac{z(s)}{\left(x_{2}-s\right)^{1-\alpha}} d s\right) \\
& \leq d\left(\frac{1}{\Gamma(\alpha)} \int_{0}^{x_{1}} \frac{z(s)}{\left(x_{1}-s\right)^{1-\alpha}} d s, \frac{1}{\Gamma(\alpha)}\right. \\
& \left.\cdot \int_{0}^{x_{1}} \frac{z(s)}{\left(x_{2}-s\right)^{1-\alpha}} d s\right)+d\left(\frac{1}{\Gamma(\alpha)}\right. \\
& \left.\cdot \int_{x_{1}}^{x_{2}} \frac{z(s)}{\left(x_{2}-s\right)^{1-\alpha}} d s, \widehat{0}\right) \\
& \leq \frac{r}{\Gamma(\alpha)} \mid \int_{0}^{x_{1}}\left(\frac{1}{\left(x_{1}-s\right)^{1-\alpha}}-\frac{1}{\left(x_{2}-s\right)^{1-\alpha}}\right) d s \\
& +\frac{r}{\Gamma(\alpha)} \int_{x_{1}}^{x_{2}} \frac{1}{\left(x_{2}-s\right)^{1-\alpha}} d s \leq \frac{2 r}{\Gamma(1+\alpha)}\left(x_{2}-x_{1}\right)^{\alpha} \\
& +\frac{r}{\Gamma(1+\alpha)}\left(x_{2}^{\alpha}-x_{1}^{\alpha}\right) .
\end{aligned}
$$

Estimating the third term of above equation similarly to above, we have

$$
\begin{aligned}
& d\left(\frac{1}{\Gamma(\alpha-\beta)} \int_{0}^{x_{1}} \frac{z(s)}{\left(x_{1}-s\right)^{1-(\alpha-\beta)}} d s, \frac{1}{\Gamma(\alpha-\beta)}\right. \\
& \left.\quad \cdot \int_{0}^{x_{2}} \frac{z(s)}{\left(x_{2}-s\right)^{1-(\alpha-\beta)}} d s\right) \leq \frac{2 r}{\Gamma(1+\alpha-\beta)}\left(x_{2}\right. \\
& \left.-x_{1}\right)^{\alpha-\beta}+\frac{r}{\Gamma(1+\alpha-\beta)}\left(x_{2}^{\alpha-\beta}-x_{1}^{\alpha-\beta}\right) .
\end{aligned}
$$

So we obtain as follows:

$$
\begin{aligned}
& d\left(T z\left(x_{1}\right), T z\left(x_{2}\right)\right) \\
& \leq L_{3}\left|x_{1}-x_{2}\right|+\frac{2 r L_{1}}{\Gamma(1+\alpha)}\left(x_{2}-x_{1}\right)^{\alpha} \\
& \quad+\frac{r L_{1}}{\Gamma(1+\alpha)}\left(x_{2}^{\alpha}-x_{1}^{\alpha}\right) \\
& \quad+\frac{2 r L_{2}}{\Gamma(1+\alpha-\beta)}\left(x_{2}-x_{1}\right)^{\alpha-\beta} \\
& \quad+\frac{r L_{2}}{\Gamma(1+\alpha-\beta)}\left(x_{2}^{\alpha-\beta}-x_{1}^{\alpha-\beta}\right) .
\end{aligned}
$$

From right side of the above expression, we can see that $\left\{T u \mid u \in \bar{U}_{r}(\widehat{0})\right\}$ is equicontinuous.

By Arzelà-Ascoli theorem, $\left\{T u \mid u \in \bar{U}_{r}(\widehat{0})\right\}$ is relatively compact.

By Theorem 20 and Schauder fixed point theorem, we can see the operator $T$ has a fixed point in $\bar{U}_{r}(\widehat{0})$.

\section{Continuous Dependence on Initial Condition of Solution}

We consider the continuous dependence on initial condition of solution for proposed problem (24). Let $y_{1}\left(t, y_{1,0}\right)$, $y_{2}\left(t, y_{2,0}\right)$ are solutions with initial values $y_{1,0}, y_{2,0} \in \mathbf{R}_{\mathrm{F}}$ and $z_{1}\left(t, y_{1,0}\right), z_{2}\left(t, y_{2,0}\right)$ are solutions of corresponding integral equation.

The following theorem shows the continuous dependence on initial condition of solution.

Theorem 21. Suppose that the following conditions are satisfied:

(i) $\exists L_{1}, L_{2}>0 ; \forall x \in[0,1], \forall y_{1}, y_{2}, z_{1}, z_{2} \in C^{F}(I)$,

$$
\begin{aligned}
& d\left(f\left(x, y_{1}, z_{1}\right), f\left(x, y_{2}, z_{2}\right)\right) \\
& \quad \leq L_{1} \cdot d\left(y_{1}, y_{2}\right)+L_{2} \cdot d\left(z_{1}, z_{2}\right) .
\end{aligned}
$$

(ii) $q^{*}:=L_{1}(1 / \Gamma(\alpha+1))+L_{2} \cdot 1 / \Gamma(\alpha-\beta+1)<1$.

Then following relation holds:

$$
\begin{aligned}
& d\left(y_{1}\left(t, y_{1,0}\right), y_{2}\left(t, y_{2,0}\right)\right) \\
& \quad \leq\left(1+\frac{L_{1}}{\left(1-q^{*}\right) \Gamma(\alpha+1)}\right) d\left(y_{1,0}, y_{2,0}\right)
\end{aligned}
$$

Proof. Since $z_{1}\left(t, y_{1,0}\right), z_{2}\left(t, y_{2,0}\right)$ are solutions of the integral equation (40), we have

$$
\begin{aligned}
& z_{1}\left(x, y_{1,0}\right) \\
& \quad \equiv f\left(x, y_{1,0} \oplus I_{0+}^{\alpha} z_{1}\left(x, y_{1,0}\right), I_{0+}^{\alpha-\beta} z_{1}\left(x, y_{1,0}\right)\right), \\
& z_{2}\left(x, y_{2,0}\right) \\
& \quad \equiv f\left(x, y_{2,0} \oplus I_{0+}^{\alpha} z_{2}\left(x, y_{2,0}\right), I_{0+}^{\alpha-\beta} z_{2}\left(x, y_{2,0}\right)\right) .
\end{aligned}
$$

Now we estimate $d\left(z_{1}\left(x, y_{1,0}\right), z_{2}\left(x, y_{2,0}\right)\right)$.

$$
\begin{aligned}
& d\left(z_{1}\left(x, y_{1,0}\right), z_{2}\left(x, y_{2,0}\right)\right) \\
& \quad=d\left(f\left(x, y_{1,0} \oplus I_{0+}^{\alpha} z_{1}\left(x, y_{1,0}\right), I_{0+}^{\alpha-\beta} z_{1}\left(x, y_{1,0}\right)\right),\right. \\
& \left.f\left(x, y_{2,0} \oplus I_{0+}^{\alpha} z_{2}\left(x, y_{2,0}\right), I_{0+}^{\alpha-\beta} z_{2}\left(x, y_{2,0}\right)\right)\right) \\
& \quad \leq L_{1} d\left(y_{1,0} \oplus I_{0+}^{\alpha} z_{1}\left(x, y_{1,0}\right), y_{2,0} \oplus I_{0+}^{\alpha} z_{2}\left(x, y_{2,0}\right)\right) \\
& \quad+L_{2} d\left(I_{0+}^{\alpha-\beta} z_{1}\left(x, y_{1,0}\right), I_{0+}^{\alpha-\beta} z_{2}\left(x, y_{2,0}\right)\right) \\
& \quad \leq L_{1}\left(d\left(y_{1,0}, y_{2,0}\right)\right. \\
& \left.\quad+d\left(I_{0+}^{\alpha} z_{1}\left(x, y_{1,0}\right), I_{0+}^{\alpha} z_{2}\left(x, y_{2,0}\right)\right)\right) \\
& \quad+L_{2} d\left(I_{0+}^{\alpha-\beta} z_{1}\left(x, y_{1,0}\right), I_{0+}^{\alpha-\beta} z_{2}\left(x, y_{2,0}\right)\right)
\end{aligned}
$$


By (iii) of Lemma 14, we have

$$
\begin{aligned}
\leq & L_{1}\left(d\left(y_{1,0}, y_{2,0}\right)+I_{0+}^{\alpha} d\left(z_{1}\left(x, y_{1,0}\right), z_{2}\left(x, y_{2,0}\right)\right)\right) \\
& +L_{2} I_{0+}^{\alpha-\beta} d\left(z_{1}\left(x, y_{1,0}\right), z_{2}\left(x, y_{2,0}\right)\right) \\
\leq & L_{1} d\left(y_{1,0}, y_{2,0}\right)+\frac{L_{1}}{\Gamma(\alpha+1)} d^{*}\left(z_{1}, z_{2}\right) \\
& +\frac{L_{2}}{\Gamma(\alpha-\beta+1)} d^{*}\left(z_{1}, z_{2}\right) \\
= & L_{1} d\left(y_{1,0}, y_{2,0}\right)+q^{*} d^{*}\left(z_{1}, z_{2}\right) .
\end{aligned}
$$

Consequently, we get

$$
\begin{aligned}
& \max _{x \in(0,1]} d\left(z_{1}\left(x, y_{1,0}\right), z_{2}\left(x, y_{2,0}\right)\right)=d^{*}\left(z_{1}, z_{2}\right) \\
& \quad \leq L_{1} d\left(y_{1,0}, y_{2,0}\right)+q^{*} d^{*}\left(z_{1}, z_{2}\right) \\
& d^{*}\left(z_{1}, z_{2}\right) \leq \frac{L_{1}}{1-q^{*}} d\left(y_{1,0}, y_{2,0}\right) .
\end{aligned}
$$

Next we estimate $d\left(y_{1}\left(t, y_{1,0}\right), y_{2}\left(t, y_{2,0}\right)\right)$.

$$
\begin{aligned}
& d\left(y_{1}\left(t, y_{1,0}\right), y_{2}\left(t, y_{2,0}\right)\right)=d\left(y_{1,0} \oplus \frac{1}{\Gamma(\alpha)}\right. \\
& \left.\cdot \int_{0}^{x} \frac{z_{1}\left(s, y_{1,0}\right)}{(x-s)^{1-\alpha}} d s, y_{2,0} \oplus \frac{1}{\Gamma(\alpha)} \int_{0}^{x} \frac{z_{2}\left(s, y_{1,0}\right)}{(x-s)^{1-\alpha}} d s\right) \\
& \leq d\left(y_{1,0}, y_{2,0}\right)+d\left(\frac{1}{\Gamma(\alpha)} \int_{0}^{x} \frac{z_{1}\left(s, y_{1,0}\right)}{(x-s)^{1-\alpha}} d s, \frac{1}{\Gamma(\alpha)}\right. \\
& \left.\quad \cdot \int_{0}^{x} \frac{z_{2}\left(s, y_{1,0}\right)}{(x-s)^{1-\alpha}} d s\right)
\end{aligned}
$$

By (iii) of Lemma 14, we have

$$
\begin{aligned}
& \leq d\left(y_{1,0}, y_{2,0}\right) \\
& \quad+\frac{1}{\Gamma(\alpha)} \int_{0}^{x} \frac{d\left(z_{1}\left(s, y_{1,0}\right), z_{2}\left(s, y_{1,0}\right)\right)}{(x-s)^{1-\alpha}} d s \\
& \leq d\left(y_{1,0}, y_{2,0}\right)+\frac{1}{\Gamma(\alpha+1)} d^{*}\left(z_{1}, z_{2}\right) .
\end{aligned}
$$

By (81), we obtain

$$
\begin{aligned}
& d\left(y_{1}\left(t, y_{1,0}\right), y_{2}\left(t, y_{2,0}\right)\right) \\
& \quad \leq\left(1+\frac{L_{1}}{\left(1-q^{*}\right) \Gamma(\alpha+1)}\right) d\left(y_{1,0}, y_{2,0}\right) .
\end{aligned}
$$

\section{Conclusions}

We obtained the uniqueness results by employing contraction mapping principle and the existence results by using
Schauder fixed point theorem for the solution of the fuzzy multiterm fractional differential equation involving Caputotype fuzzy fractional derivative of order $0<\alpha<1$ on complete metric space of continuous fuzzy number value functions. We also established the continuous dependence of solution on its initial condition. Our results can be expanded to the case in which the right side of equation involves more than two derivative terms.

\section{Data Availability}

No data were used to support this study.

\section{Conflicts of Interest}

The authors declare that there are no conflicts of interest regarding the publication of this paper.

\section{References}

[1] S. S. Mansouri, M. Gachpazan, and O. S. Fard, "Existence, uniqueness and stability of fuzzy fractional differential equations with local Lipschitz and linear growth conditions," Advances in Difference Equations, vol. 2017, p. 240, 2017.

[2] A. Rivaz, O. S. Fard, and T. A. Bidgoli, "On the existence and uniqueness of solutions for fuzzy fractional differential equations," Tbilisi Mathematical Journal, vol. 10, no. 1, pp. 197205, 2017.

[3] R. P. Agarwal, V. Lakshmikantham, and J. J. Nieto, "On the concept of solution for fractional differential equations with uncertainty," Nonlinear Analysis: Theory, Methods \& Applications, vol. 72, no. 6, pp. 2859-2862, 2010.

[4] S. Arshad and V. Lupulescu, "On the fractional differential equations with uncertainty," Nonlinear Analysis: Theory, Methods \& Applications, vol. 74, no. 11, pp. 3685-3693, 2011.

[5] A. Khastan, J. J. Nieto, and R. Rodríguez-López, "Periodic boundary value problems for first-order linear differential equations with uncertainty under generalized differentiability," Information Sciences, vol. 222, pp. 544-558, 2013.

[6] A. Khastan, J. J. Nieto, and R. Rodríguez-López, "Schauder fixed-point theorem in semilinear spaces and its application to fractional differential equations with uncertainty," Fixed Point Theory and Applications, vol. 2014, article 21, 14 pages, 2014.

[7] A. Khastan, J. J. Nieto, and R. Rodríguez-López, "Fuzzy delay differential equations under generalized differentiability," Information Sciences, vol. 275, pp. 145-167, 2014.

[8] S. Salahshour, A. Ahmadian, F. Ismail, and D. Baleanu, "A fractional derivative with non-singular kernel for interval-valued functions under uncertainty," Optik - International Journal for Light and Electron Optics, vol. 130, pp. 273-286, 2017.

[9] S. Salahshour, A. Ahmadian, S. Abbasbandy, and D. Baleanu, " $M$-fractional derivative under interval uncertainty: theory, properties and applications," Chaos, Solitons \& Fractals, vol. 117, pp. 84-93, 2018.

[10] V. Lupulescu, "Fractional calculus for interval-valued functions," Fuzzy Sets and Systems, vol. 265, pp. 63-85, 2015.

[11] M. T. Malinowski, "Random fuzzy fractional integral equations-theoretical foundations," Fuzzy Sets and Systems, vol. 265, pp. 39-62, 2015. 
[12] T. Allahviranloo, Z. Gouyandeh, A. Armand, and A. Hasanoglu, "On fuzzy solutions for heat equation based on generalized Hukuhara differentiability," Fuzzy Sets and Systems, vol. 265, pp. 1-23, 2015.

[13] S. Salahshour, T. Allahviranloo, and S. Abbasbandy, "Solving fuzzy fractional differential equations by fuzzy Laplace transforms," Communications in Nonlinear Science and Numerical Simulation, vol. 17, no. 3, pp. 1372-1381, 2012.

[14] H. Wang, "Monotone iterative method for boundary value problems of fuzzy differential equations," Journal of Intelligent \& Fuzzy Systems: Applications in Engineering and Technology, vol. 30, no. 2, pp. 831-843, 2016.

[15] S. Salahshour, A. Ahmadian, and D. Baleanu, "Variation of constant formula for the solution of interval differential equations of non-integer order," The European Physical Journal Special Topics, vol. 226, no. 16-18, pp. 3501-3512, 2017.

[16] A. Ahmadian, S. Salahshour, and C. S. Chan, "Fractional differential systems: a fuzzy solution based on operational matrix of shifted chebyshev polynomials and its applications," IEEE Transactions on Fuzzy Systems, vol. 25, no. 1, pp. 218-236, 2017.

[17] K. Sin, M. Chen, H. Choi, and K. Ri, "Fractional Jacobi operational matrix for solving Fuzzy fractional differential equation," Journal of Intelligent \& Fuzzy Systems: Applications in Engineering and Technology, vol. 33, no. 2, pp. 1041-1052, 2017.

[18] K. Sin, C. Minghao, W. Chong et al., "Application of a spectral method to fractional Differential Equations under uncertainty," Journal of Intelligent \& Fuzzy Systems: Applications in Engineering and Technology, vol. 35, no. 4, pp. 4821-4835, 2018.

[19] M. Mazandarani and A. V. Kamyad, "Modified fractional Euler method for solving fuzzy fractional initial value problem," Communications in Nonlinear Science and Numerical Simulation, vol. 18, no. 1, pp. 12-21, 2013.

[20] A. Ahmadian, F. Ismail, S. Salahshour, D. Baleanu, and F. Ghaemi, "Uncertain viscoelastic models with fractional order: a new spectral tau method to study the numerical simulations of the solution," Communications in Nonlinear Science and Numerical Simulation, vol. 53, pp. 44-64, 2017.

[21] A. Ahmadian, H. S. Chan, S. Salahshour, and V. Vaitheeswaran, "FTFBE: A numerical approximation for fuzzy time-fractional Bloch equation," in Proceedings of the 2014 IEEE International Conference on Fuzzy Systems, FUZZ-IEEE 2014, pp. 418-423, July 2014.

[22] S. Salahshour, T. Allahviranloo, S. Abbasbandy et al., "Existence and uniqueness results for fractional differential equations with uncertainty," Advances in Difference Equations, vol. 2012, article 112, 12 pages, 2012.

[23] Z.-P. Yang, T.-Z. Xu, and M. Qi, “The Cauchy problem for quaternion fuzzy fractional differential equations," Journal of Intelligent \& Fuzzy Systems: Applications in Engineering and Technology, vol. 29, no. 1, pp. 451-461, 2015.

[24] V. H. Ngo, "Fuzzy fractional functional integral and differential equations," Fuzzy Sets and Systems, vol. 280, pp. 58-90, 2015.

[25] P. Prakash, J. J. Nieto, S. Senthilvelavan, and G. S. Priya, "Fuzzy fractional initial value problem," Journal of Intelligent \& Fuzzy Systems: Applications in Engineering and Technology, vol. 28, no. 6, pp. 2691-2704, 2015.

[26] A. Souahi, A. Guezane-Lakoud, and A. Hitta, "On the existence and uniqueness for high order fuzzy fractional differential equations with uncertainty," Advances in Fuzzy Systems, vol. 2016, Article ID 5246430, 9 pages, 2016.
[27] H. V. Ngo, V. Ho, and T. M. Duc, "Fuzzy fractional differential equations under CaputoKatugampola fractional derivative approach," Fuzzy Sets and Systems, 2018.

[28] H. V. Ngo, V. Lupulescu, and D. O'Regan, "A note on initial value problems for fractional fuzzy differential equations," Fuzzy Sets and Systems, vol. 347, pp. 54-69, 2018.

[29] B. Bede and S. G. Gal, "Generalizations of the differentiability of fuzzy-number-valued functions with applications to fuzzy differential equations," Fuzzy Sets and Systems, vol. 151, no. 3, pp. 581-599, 2005. 


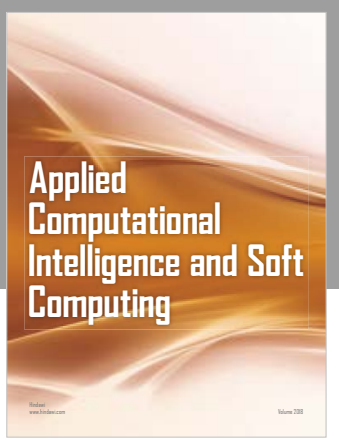

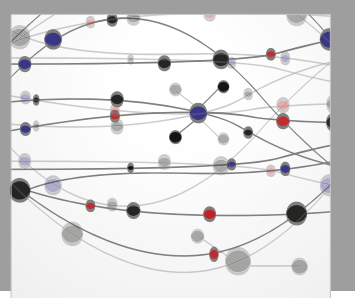

The Scientific World Journal
Submit your manuscripts at

Computing
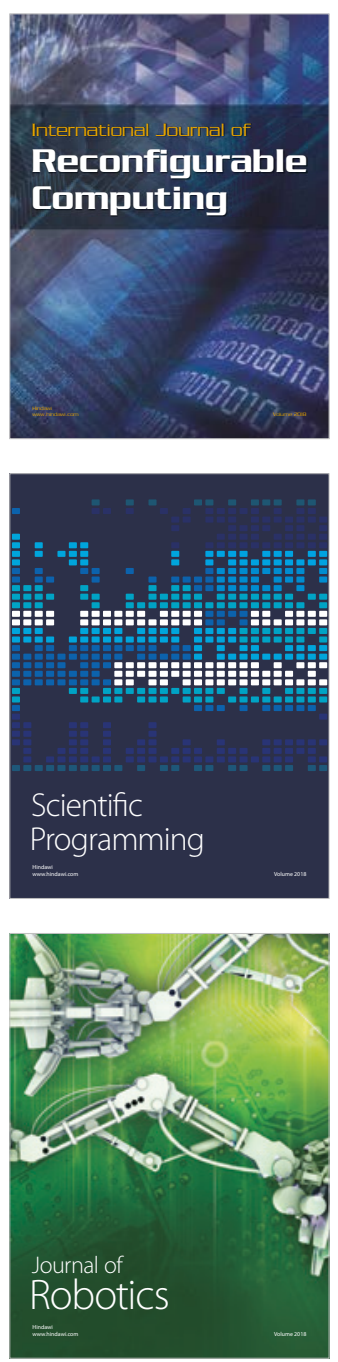

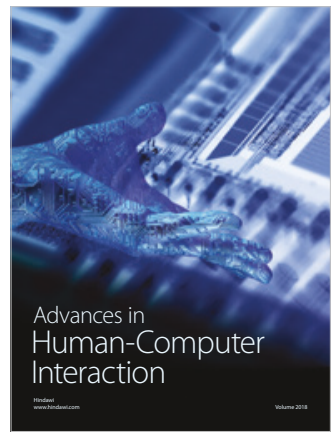

Human-Compute

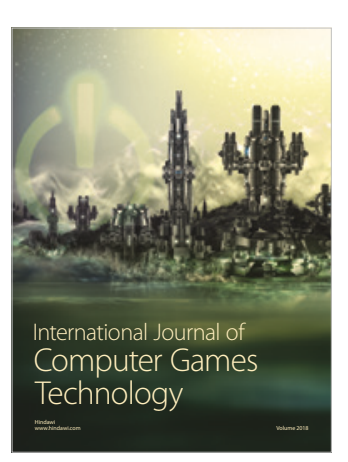

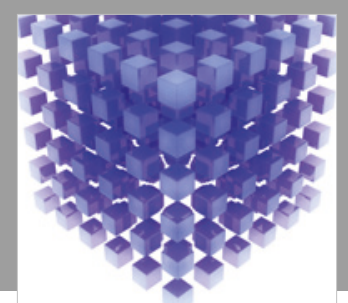

Mathematical Problems in Engineering

\section{Engincering}
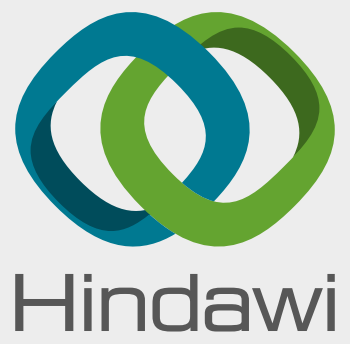

www.hindawi.com
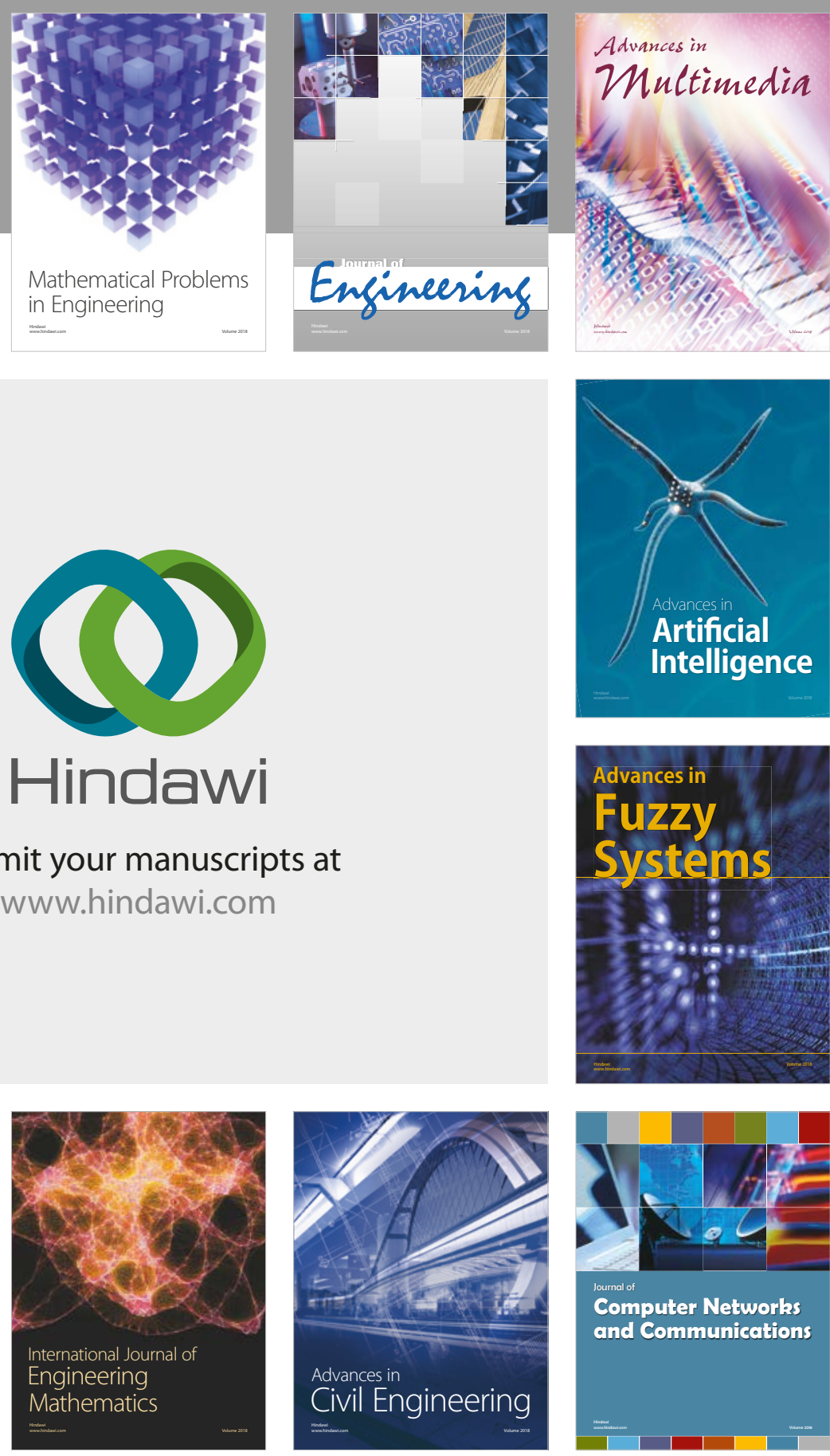

Computer Networks and Communications

Multimedia
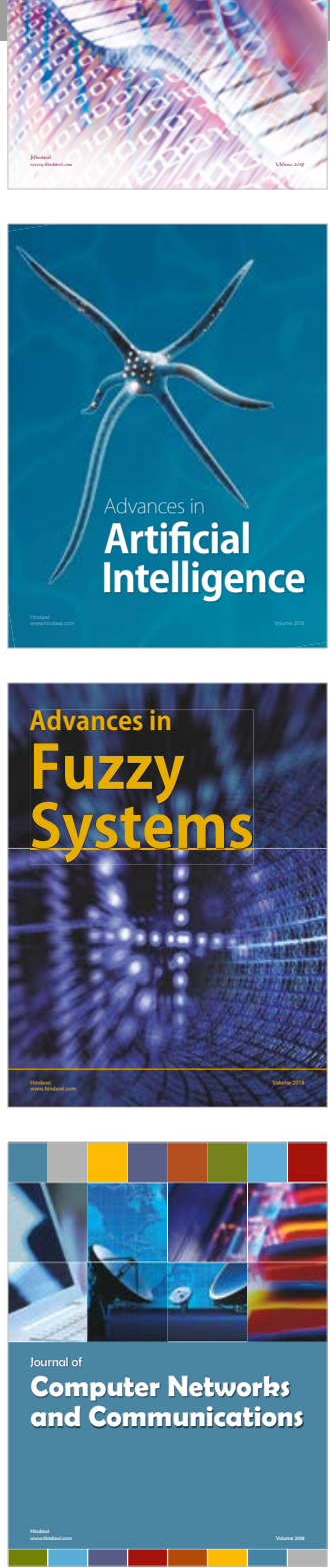

Advances in

Modelling \&

Simulation

in Engineering

interaction

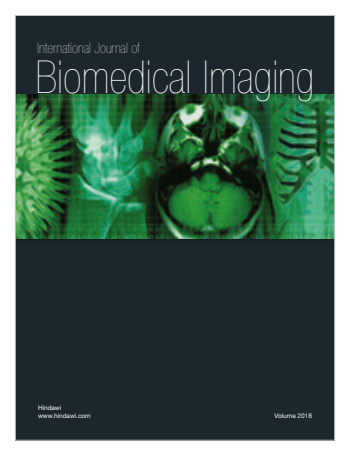

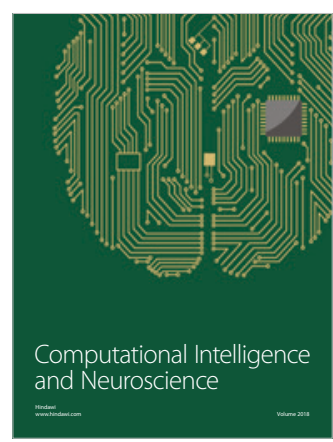

